\title{
Fabrication of liquid crystalline periodic waveguiding structures by means of the photo-polymerization process
}

\author{
B. Turowski, K. Rutkowska \\ Faculty of Physics, Warsaw University of Technology, Koszykowa 75, 00-662 Warszawa, Poland \\ Received September 25, 2017; accepted September 28, 2017; published September 30, 2017
}

\begin{abstract}
Photonic structures in the form of waveguide channels, with periodic spatial changes in the refractive index of the wavelength scale, are typically manufactured, based on a well-developed silica and gallium arsenide technology [1] as well as high-precision lithography. The main disadvantage related to their practical application is that their optical properties cannot be altered after fabrication. Due to this fact, liquid crystalline periodic waveguiding structures, manufactured by a process of photo-polymerization, have been proposed in this work. Importantly, the propagational characteristic of such photonic structures can be easily adjusted during fabrication, and then also dynamically tuned, e.g. by the application of external voltage, as presented in the present communication.
\end{abstract}

Liquid crystalline periodic waveguiding structures proposed in this work have been fabricated by a process of photo-polymerization. Polymer stabilized liquid crystals (LCs) in the nematic phase are constantly considered as perfect candidates for functional elements (including waveguides) for integrated optical circuits [2]. The process requires the proper concentration of a monomer to be added to the liquid crystalline material [3], as well as proper UV irradiation conditions to be found empirically. The latter is particularly important in obtaining high-quality periodic photonic structures in the liquid crystalline material. For this reason, UV illumination conditions have been carefully studied in this work. Later on, light beam propagation was observed in the obtained structure of the best quality.

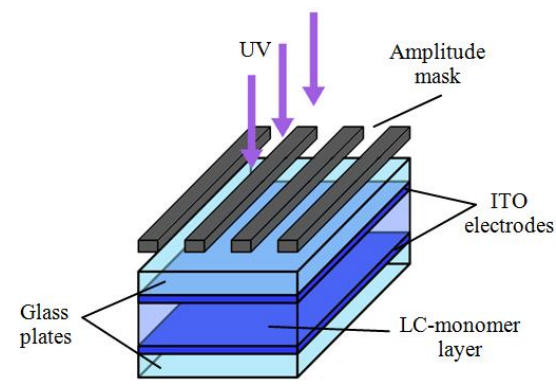

Fig. 1. Liquid crystal cell under UV irradiation through a periodic mask in the photo-polymerization process to create periodic waveguiding structures.

Applied here to define the regions with a different effective refractive index, the photo-polymerization process (taking place in an LC cell and schematically presented in Fig. 1) takes advantage of polymerization of an LC-monomer mixture subjected to UV light. In this work, a 6CHBT liquid crystal [4] with an addition of 4.2 wt. \% of RM257 monomer has been applied. The latter is widely used for liquid crystal stabilization [5, 6] and for e.g. microgratings fabrication [7].

A fifty-fifty comb-shaped amplitude chromium mask was placed on a sandwich-like cell filled with an LC-monomer mixture during the photo-polymerization process, as presented in Fig. 1. Mechanical rubbing, performed in an initial stage of LC sample preparation, provides the planar orientation of liquid crystalline molecules in the entire cell when it is not subjected to an external electric field. A suitably high voltage applied to an LC cell allows for the molecules to be reoriented up to a homeotropic arrangement. Due to the photo-polymerization process performed in this state, the LC molecular orientation in an unmasked area can be halted even after a biasing voltage is turned off. It allows to obtain regions with a different effective refractive index for a linearly polarized light beam (while the areas of planar and homeotropic molecular arrangement are present in the LC layer), as well as tune an index contrast between these two regions. The latter can be achieved thanks to the electrically-driven LC molecular reorientation in the region that was masked during UV irradiation.

As already mentioned, the proper concentration of a monomer in the LC material was ensured. All components of the liquid crystalline material and monomer mixture analyzed here were prepared with milligram-accuracy. The mixture was obtained in the following process: a small amount (less than 1 wt. \% of the whole LCmonomer mixture) of the photo-activator (PPD) was added to a precisely weighed amount of the RM257 monomer (both in a powder form), placed in a glass phial. Then, such powder components were added to the right amount of the liquid crystalline material in an isotropic phase (which is above $43^{\circ} \mathrm{C}$ for 6CHBT LC [4]) to obtain the final mixture with 4.2 wt. \% of the RM257 monomer. The concentration of RM 257 in the LC monomer mixture, applied to obtained periodic waveguiding structures in an LC material, was previously studied in Ref. [8]. Later on, the glass phial was shaken mechanically with a relatively low frequency (around 1000 shakes per second) for 1 minute in order to remove bigger inhomogeneities and then placed in a $100 \mathrm{kHz}$ ultrasonic bath for 15 minutes in $55^{\circ} \mathrm{C}$ to obtain a fully uniform mixture. Eventually, the LC 
cells were infiltrated with an LC-monomer mixture at the same temperature to keep the LC in an isotropic phase. Then, UV irradiations of so prepared samples were performed at room temperature. The photos of fabricated structures, placed between crossed-polarizers, were taken with a high-resolution digital microscope (as shown in Fig. 4).

The initial tests of UV irradiation allowed to determinate that the power density of UV light and applied bias should be kept as $90 \mathrm{~mW} / \mathrm{cm}^{2}$ and $140 \mathrm{~V}$, respectively. The energy of UV light used for the photopolymerization process was adjusted by changing the duration of single UV irradiation and the number of repetitions. The time intervals between each irradiation were also attuned so that thermal effects did not affect the LC molecular arrangement in the LC cell during UV illumination.

LC structures were fabricated in two different experimental setups. The first one, shown in Fig. 2, was used to test the influence of UV irradiation and to determine roughly the illumination conditions. The second experimental setup (sketched in Fig. 3) was applied for precise specification of UV irradiation time, number of repetitions and time intervals in between (as given for the example in Table 1).

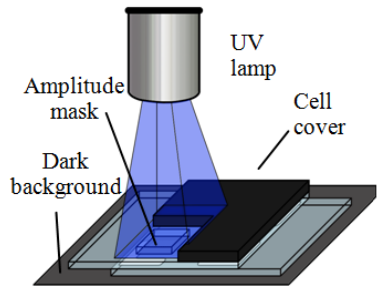

Fig. 2. Experimental setup for initial tests of UV irradiation.

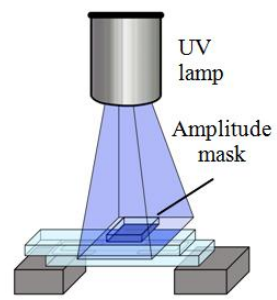

Fig. 3. Modified experimental setup for precise determination of UV illumination conditions.
The cell cover present in the first experimental setup (Fig. 2) was applied to prevent the polymerization process from taking place over the whole area of the LC cell and thus to allow sectional irradiation of the sample. The dark background in this setup was additionally used to reduce backscattering of UV light from the surface on which the cell was placed. After further modifications of the experimental conditions, the LC cell was elevated in order to further reduce the influence of backscatter UV light on the fabricated structure (see Fig. 3).

It is worth noting that more than 30 different samples were fabricated during the studies performed within this work. The irradiation parameters for the best obtained structures are listed in Table 1, while the photos of their top views (taken in transmitted light and with crosspolarizers) are presented in Fig. 4. UV irradiations, whose results are shown in Fig. $4(a, b)$, were performed to test what happens at higher energy of UV light while the total illumination time is maintained (i.e. the number of UV irradiation repetitions multiplied by a single irradiation duration). When they are compared with the results presented in Fig. 4 (c, d), it may be noticed that even though UV light energy is the same, there are too few repetitions of UV irradiation for the RM257 monomer to fully polymerize. As a result, the molecules do not maintain a desirable orientation, as visible in Fig. 4 (b). In both cases (i.e. for Fig. 4 (a, b) and Fig. 4 (c, d)) the obtained strips of periodic structures are sharp and welldefined but in the case presented in Fig. 4 (c, d) the thermal effect still influences the molecular arrangement in the masked area (which should remain planar in a perfect situation). It suggests that the time interval between irradiation acts should be increased, as in the case shown in Fig. 4 (e, f).

Table 1. UV irradiation parameters for three different periodic waveguiding structures obtained in an LC-monomer mixture.

\begin{tabular}{|c|c|c|c|}
\hline & $\begin{array}{c}\text { Number of UV } \\
\text { irradiation } \\
\text { repetitions }\end{array}$ & $\begin{array}{c}\text { Single } \\
\text { irradiation } \\
\text { duration [s] }\end{array}$ & $\begin{array}{c}\text { Time interval } \\
\text { between single } \\
\text { irradiations [s] }\end{array}$ \\
\hline Fig. 4 (a, b) & 4 & 0.60 & 1200 \\
\hline Fig. 4 (c, d) & 6 & 0.40 & 900 \\
\hline Fig. 4 (e, f) & 6 & 0.45 & 1200 \\
\hline
\end{tabular}
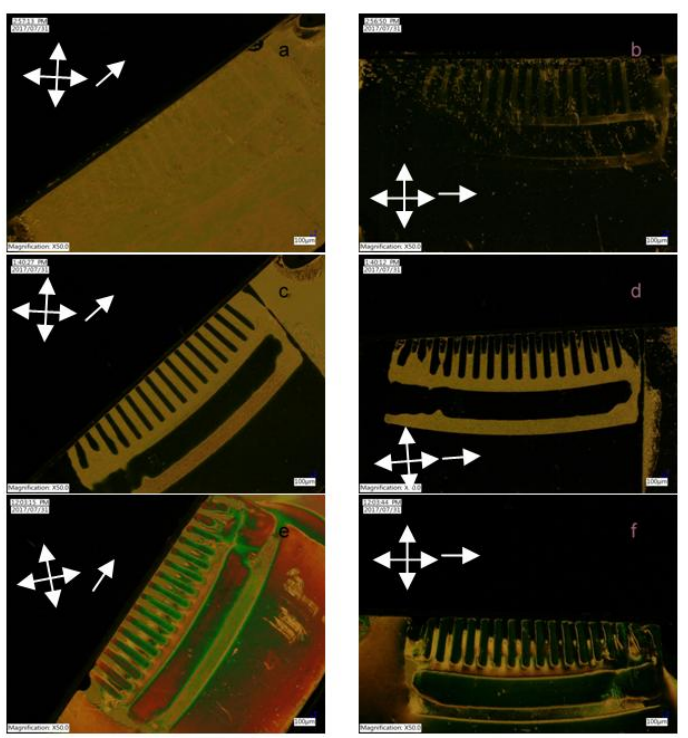

Fig. 4. Results of UV irradiation procedures leading to the creation of well-defined waveguiding channels in a liquid crystalline material. Irradiation parameters are specified in Table 1. The single arrows marked in the graphs represent the initial (planar) molecular orientation in the LC cell, while the double ones correspond to the crossed-polarizers axes.

After all trials and adjustments performed, it was eventually judged that the best obtained structure was this one shown in Fig. 4 (e, f). Even though the obtained stripes, defining the regions with a different LC molecular orientation, are slightly inflated, they are sharp and well- 
defined. This structure was further examined in a simple experimental setup in which the LC cell was placed directly under a CCD camera allowing for the light scattered above the cell to be registered. A light beam from the laser diode emitting in red was focused on the input edge of the LC cell with a long-working distance microscope objective. A polarizer was used to set the exact direction of linear polarization of the light beam at the input of the LC cell. Specifically, TM- and TE-like polarizations (which are vertical and horizontal with respect to the LC cell base/glass plane, respectively) were used in the tests. The position of the camera, as well as the input microscope objective might be precisely changed by micrometric screws in three directions. The cell was placed on the surface, which placement could be similarly adjusted along three mutually perpendicular axes. In tests, an electrical voltage (above 140V) was applied to the LC cell to observe its influence on light propagation in a periodic waveguiding structure.

Figure 5 presents light beam propagation in an area which was masked during UV irradiation. The white symbols indicated the direction of a molecular director in this area, while theyellow ones define the direction of linear polarization of a light beam launched into the LC cell.
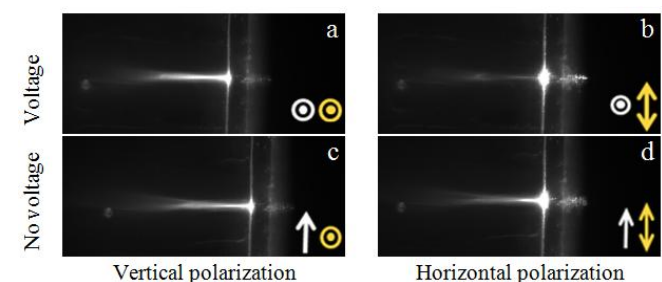

Fig. 5. Light beams launched into the masked area of the sample with $(\mathrm{a}, \mathrm{b})$ and without (c, d) electric voltage applied.

Applied voltage changes the molecular arrangement in the area of with an initial planar orientation to a homeotropic one. By comparing the photos shown in Fig. 5 (a, c), it is visible that the light beam is slightly less diverging in the first photo (i.e. when the electric bias is on). It is due to the fact that after molecular reorientation, the effective refractive index for a vertically polarized light beam is extraordinary and the light beam is better confined in this area. In the photo shown in Fig. 5 (c) the light beam propagates in the region with an ordinary effective refractive index and it spreads wider. Analogous behaviour can be observed for the light beam of horizontal polarization (see Fig. 5 (b, d)).

When launching a light beam into the area which was unmasked during UV irradiation (as demonstrated in Fig. $6)$, the application of an electric voltage should not change much the way in which the light propagates. It is caused by the fact that liquid crystal molecules maintain their orientation while cross-linked by the polymer. The comparison of the photos shown in Fig. 6 proves the above statement. In both pairs of analyzed cases, i.e. for situations shown in Fig. $6(\mathrm{a}, \mathrm{c})$ and $6(\mathrm{~b}, \mathrm{~d})$ the light beams propagate in the same way indecently on applied voltage (the differences in the beam profiles are marginal). The light beam of vertical polarization is better confined as it propagates in the region with an extraordinary effective refractive index.
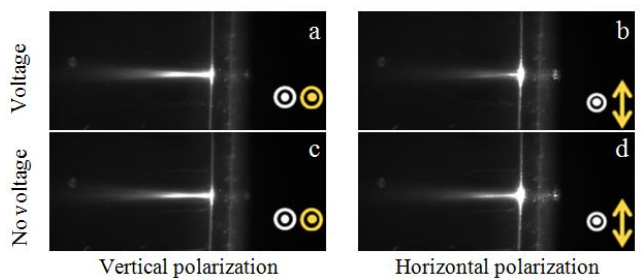

Fig. 6. Light beams introduced to the unmasked area with (a, b) and without (c, d) applied voltage.

In conclusion, the main goal of this work was to study the possibility of fabricating liquid crystalline periodic waveguiding structures by a process of photopolymerization. It was proven that the optical properties of such structures may be altered after their fabrication. It is a huge advantage in comparison to periodic waveguiding structures manufactured in other materials. Concluding the fabrication procedure, one can state that the photo-polymerization process proposed here is easy to perform but there are several important aspects that should be taken into consideration, namely the composition of an LC-monomer mixture, UV light energy, time intervals between successive irradiations. All of these factors must be precisely defined, as even a small difference might lead to a photonic structure of unsatisfactory quality. In the experimental tests performed, a different way of light beam propagation was observed depending on the area of the structure into which the light beam was launched, and the direction of linear polarization of the light beam. It was also observed, that the applied voltage may change the way that the light beam propagates in the periodic waveguiding structure under consideration, as it was anticipated.

The authors would like to acknowledge the support from the Polish National Science Centre under the grant no. DEC-2013/11/B/ST7/04330.

\section{References}

[1] M.J. Ablowitz, Z.H. Musslimani, Phys. D: Nonlin. Phenom. 184(1), 276 (2003).

[2] L. Vicari, Optical applications of liquid crystals (CRC press 2016).

[3] J. Yan, S.-T. Wu, J. Display Technol. 7, 9 (2011).

[4] J. Schirmer et al., Mol. Cryst. Liquid Cryst. 307, 17 (1997).

[5] D. Xu et al., Appl. Phys. Lett. 105, 8 (2014).

[6] I. Dierking et al., Soft Matter 8, 4355 (2012).

[7] K. Kang, L.C. Chien, S. Sprunt, Liquid Crystals 29, 9 (2002).

[8] K.A. Rutkowska, M. Chychłowski, U.A. Laudyn, Proc. SPIE 10325, 1032506 (2017). 